\title{
On the nature of Fermi Golden Rule for open quantum systems
}

\author{
Jan Dereziński ${ }^{1}$ and Vojkan Jakšićc ${ }^{2}$ \\ ${ }^{1}$ Department of Mathematical Methods in Physics \\ Warsaw University \\ Hoża 74, 00-682, Warszawa, Poland \\ ${ }^{2}$ Department of Mathematics and Statistics \\ McGill University \\ 805 Sherbrooke Street West \\ Montreal, QC, H3A 2K6, Canada
}

July 30,2003

Dedicated to Elliot Lieb on the occasion of his 70th birthday

\begin{abstract}
We consider a general class of models consisting of a small quantum system $\mathcal{S}$ interacting with a reservoir $\mathcal{R}$. We compare three applications of 2 nd order perturbation theory (the Fermi Golden Rule) to the study of such models: (1) the van Hove (weak coupling) limit for the dynamics reduced to $\mathcal{S}$; $(2)$ the Fermi Golden Rule applied to the Liouvillean - an argument that was used in recent papers on the return to equilibrium; (3) the Fermi Golden Rule applied to the so-called CLiouvillean. These three applications lead to three Level Shift Operators. As our main result, we prove that if the reservoir $\mathcal{R}$ is thermal (if it satisfies the KMS condition), then the Level Shift Operator obtained in (1) (often called the Davies generator) and the Level Shift Operator constructed in (2) are connected by a similarity transformation. We also show that the Davies generator coincides with the Level Shift Operator obtained in (3) for a general $\mathcal{R}$.
\end{abstract}




\section{Introduction}

In his 1949 Chigaco lecture notes [F], Fermi called the formulas for the 2nd order perturbative calculations of energy levels the Golden Rule. There exists a number of mathematically rigorous implementations of the Fermi Golden Rule (FGR). One of them is the so-called van Hove (or weak coupling) limit.

To describe the general structure of the van Hove limit, consider a family of operators $L_{\lambda}:=L_{0}+\lambda Q$. Let $P$ be a projection commuting with the unperturbed operator $L_{0}$ satisfying $P Q P=0$. Under appropriate assumptions [Da1, Da3], one can show that there exists an operator $\Gamma$ such that

$$
\lim _{\lambda \rightarrow 0} P \mathrm{e}^{-\mathrm{i} t L_{0} / \lambda^{2}} \mathrm{e}^{\mathrm{i} t L_{\lambda} / \lambda^{2}} P=\mathrm{e}^{\mathrm{i} t \Gamma} .
$$

We will call $\Gamma$ the Level Shift Operator (LSO).

In the literature one can find other rigorous forms of FGR. They usually express the idea that LSO describes the shift of eigenvalues and resonances at the 2nd order of perturbation theory. Some of these applications are discussed in [DJ2, DJ3]. For shortness, in this note we will restrict ourselves to the dynamical form of FGR - the van Hove limit.

There exist numerous papers studying a "small quantum system $\mathcal{S}$ interacting with a reservoir $\mathcal{R}$ ". In many of them the Fermi Golden Rule plays a central role. Among these applications of FGR to the study of $\mathcal{S}+\mathcal{R}$ we would like to distinguish the following 2 types:

(1) Van Hove limit for the reduced dynamics. We assume that the reservoir is initially in a stationary state for the unperturbed dynamics. We look at the evolution of observables of the small system. One can then show that under mild conditions the reduced dynamics in the van Hove limit is a completely positive semigroup [Da1, LeSp]. The operator $\Gamma$ obtained in this way (the generator of this semigroup) is often called the Davies generator. This construction is regarded as an example of how irreversible behavior can emerge from a reversible Hamiltonian dynamics.

(2) Fermi Golden Rule used in recent works on the return to equilibrium. The main goal of a number of recent papers [DJ2, BFS, JP1, M] is to prove that if the reservoir is in thermal state, then the coupled system $\mathcal{S}+\mathcal{R}$ has only one normal stationary state. This problem can be reformulated into a question about point spectrum of a certain naturally defined self-adjoint operator - the Liouvillean. An argument based on FGR leads to an appropriate LSO. Analysis of this LSO is the key step in the proof of a number of results related to the return to equilibrium [DJ2].

Let us stress that both in (1) and (2) we consider the same physical system $\mathcal{S}+\mathcal{R}$. Nevertheless, these two applications are quite different. 
The difference that is visible at the first sight is that in (1) we use the van Hove limit, whereas in (2) we use the spectral form of FGR. This difference is due to our physical motivation. Mathematically, one can also consider the van Hove limit for the Liouvillean, even though to our knowledge it does not have a clear physical significance.

The more important difference is that in (1) and (2) $P$ and $L_{\lambda}$ are different mathematical objects. In (1) $\exp \left(\mathrm{it} L_{\lambda}\right)$ is the Heisenberg dynamics of the algebra of observables, whereas in (2) $L_{\lambda}$ is the so-called standard Liouvillean. In (1) $P$ is the conditional expectation onto the observables of $\mathcal{S}$, whereas in (2) $P$ is the orthogonal projection onto the vacuum sector. The LSO obtained in (1) is different from the LSO obtained in (2). In particular, the two LSO's have different spectra. Note, however, that both LSO's act on the same space: the space of matrices describing the observables of the small systems $\mathcal{S}$.

The main result of our paper is the proof of the following fact: if the reservoir is in thermal equlibrium, then the two LSO's are related by a similarity transformation. Thus, in particular, in the thermal case, they are isospectral.

Our result is an example of special properties enjoyed by thermal equilibrium states [BR2]. In order to formulate it we need to use some (relatively few) concepts belonging to the area of operator algebras. In particular, the fact that the reservoir is in thermal equilibrium is expressed by the KMS condition of the reservoir state wrt the unperturbed dynamics.

In this note we also consider a 3rd application of the Fermi Golden Rule to the study of small systems coupled to a reservoir. In this application the main object is the so-called C-Liouvillean introduced in [JP5]. We show that this application is essentially equivalent to (1).

Acknowledgment. We are grateful to H. Spohn and C.-A. Pillet useful discussions. A part of this work has been done during the visit of J.D. to the McGill University, which was supported by NSERC, and during the visit to Aarhus University supported by MaPhySto funded by the Danish National Research Foundation. His research was also partly supported by the Postdoctoral Training Program HPRN-CT-2002-0277 and by KBN (grants SPUB127 and 2 P03A 027 25). The research of V.J. was partly supported by NSERC.

\section{Small quantum system interacting with a reservoir}

Consider a small quantum system $\mathcal{S}$ interacting with a reservoir $\mathcal{R}$. The Hilbert space of the system $\mathcal{S}$ is $\mathcal{K}$ and its Hamiltonian is a self-adjoint operator $K$. Its algebra of observables is $\mathcal{B}(\mathcal{K})$, the Banach space of all bounded operators on $\mathcal{K}$. Throughout the paper we will assume that $\operatorname{dim} \mathcal{K}<\infty$.

The system $\mathcal{R}$ is described by a $W^{*}$-dynamical system $\left(\mathfrak{M}_{\mathcal{R}}, \tau_{\mathcal{R}}\right)$. We assume that

$\mathfrak{M}_{\mathcal{R}}$ is given in the standard form on the Hilbert space $\mathcal{H}_{\mathcal{R}}$, and we denote by $\mathcal{H}_{\mathcal{R}}^{+}, J_{\mathcal{R}}$, and $L_{\mathcal{R}}$ the corresponding natural cone, modular conjugation, and standard Liouvillean. 
We also assume that $\left(\mathfrak{M}_{\mathcal{R}}, \tau_{\mathcal{R}}\right)$ has a distingushed normal stationary state and we denote by $\Omega_{\mathcal{R}}$ its (unique) vector representative in $\left.\mathcal{H}_{\mathcal{R}}^{+} . \mid \Omega_{\mathcal{R}}\right)\left(\Omega_{\mathcal{R}} \mid\right.$ denotes projection on $\Omega_{\mathcal{R}}$.

The coupled system $\mathcal{S}+\mathcal{R}$ is described as follows. Its algebra of observables is

$$
\mathfrak{M}=\mathcal{B}(\mathcal{K}) \otimes \mathfrak{M}_{\mathcal{R}},
$$

and its free $W^{*}$-dynamics is

$$
\tau_{0}^{t}(A)=\mathrm{e}^{\mathrm{i} t L_{0}^{\text {semi }}} A \mathrm{e}^{-\mathrm{i} t L_{0}^{\text {semi }}}, \quad A \in \mathfrak{M},
$$

where

$$
L_{0}^{\text {semi }}=K \otimes 1+1 \otimes L_{\mathcal{R}} .
$$

Let $V \in \mathfrak{M}$ be a selfadjoint perturbation and $\lambda$ a real parameter. The assumption that $V$ is bounded is made only for simplicity of exposition - the discussion of unbounded perturbations affiliated to $\mathfrak{M}$ is very similar except for a number of additional technical assumptions (see Section 5). Let

$$
\begin{gathered}
L_{\lambda}^{\text {semi }}=L_{0}^{\text {semi }}+\lambda V, \\
\tau_{\lambda}^{t}(A)=\mathrm{e}^{\mathrm{i} t_{\lambda}^{\text {semi }}} A \mathrm{e}^{-\mathrm{i} t L_{\lambda}^{\text {semi }}} .
\end{gathered}
$$

The $W^{*}$-dynamical system $\left(\mathfrak{M}, \tau_{\lambda}\right)$ describes the interacting system $\mathcal{S}+\mathcal{R}$ in the so called semistandard representation. This representation is commonly used in the literature on Markovian semigroups of open quantum systems.

Following the terminology of [DJ3], the operators $L_{0}^{\text {semi }}$ and $L_{\lambda}^{\text {semi }}$ are called the free and full semi-Liouvilleans respectively.

A typical example of the reservoir system is a free Fermi or Bose gas in thermal equilibrium at inverse temperature $\beta>0$. The reservoir may also have a composite structure and consist of $N$-subreservoirs at different temperatures (such reservoirs have been studied in the literature on non-equilibrium quantum statistical mechanics, see [JP4, JP5, LeSp, Ru]). For our purposes, it is natural to keep the reservoir system as general as possible.

The effect of the reservoir on the dynamics of $\mathcal{S}$ in the weak coupling regime ( $\lambda$ small $)$ has been subject of many studies. A traditional approach to this question has been to integrate the variables of the reservoir and follow the reduced dynamics of the small system on the Van Hove time scale $t / \lambda^{2}$. In the Van Hove weak coupling limit $\lambda \rightarrow 0$, the reduced dynamics of $\mathcal{S}$ becomes Markovian and irreversible. Its generator-often computed by a formal Fermi Golden Rule calculation - captures the basic physical processes (energy emission/absorption) of open quantum system $\mathcal{S}$ to the 2nd order of perturbation theory.

This approach can be traced back to the works of Pauli, Wigner-Weisskopf and Van Hove $[\mathrm{Pa} 1, \mathrm{~Pa} 2, \mathrm{~W}, \mathrm{VH}]$, and has become a source of many works in physics literature (see e.g. [Haa, KTH] for references and additional information). 
On the mathematical side, the first complete results concerning existence of the Van Hove limit and form of the Markovian generator were obtained by Davies [Da1, Da2]. These papers were followed by a large body of mathematical physics literature (see [GFVKS] for a review of early results). The Davies theory and early mathematical results in the theory of Markovian generators of open quantum systems are discussed in detail in the forthcoming article [DJ3].

The integration of the reservoir variables is formalized as follows. We will work in the Heisenberg picture. For $B \otimes C \in \mathcal{B}\left(\mathcal{K} \otimes \mathcal{H}_{\mathcal{R}}\right)$ let

$$
P_{\mathrm{H}}(B \otimes C)=\left(\Omega_{\mathcal{R}} \mid C \Omega_{\mathcal{R}}\right) B \otimes 1 .
$$

The map $P_{\mathrm{H}}$ uniquely extends to a projection on the Banach space $\mathcal{B}\left(\mathcal{K} \otimes \mathcal{H}_{\mathcal{R}}\right)$. We identify $\mathcal{B}(\mathcal{K})$ with $\operatorname{Ran} P_{\mathrm{H}}$ by $\mathcal{B}(\mathcal{K}) \ni B \mapsto B \otimes 1$. Obviously, for $X, B \in \mathcal{B}(\mathcal{K})$,

$$
\operatorname{Tr}_{\mathcal{K} \otimes \mathcal{H}_{\mathcal{R}}}\left(X \otimes \mid \Omega_{\mathcal{R}}\right)\left(\Omega_{\mathcal{R}} \mid \tau_{0}^{-t} \tau_{\lambda}^{t}(B \otimes 1)\right)=\operatorname{Tr}_{\mathcal{K}}\left(X P_{\mathrm{H}} \tau_{0}^{-t} \tau_{\lambda}^{t} P_{\mathrm{H}} B\right) .
$$

The maps

$$
T_{\lambda}^{t}:=P_{\mathrm{H}} \tau_{0}^{-t} \tau_{\lambda}^{t} P_{\mathrm{H}}: \mathcal{B}(\mathcal{K}) \rightarrow \mathcal{B}(\mathcal{K})
$$

describe the reduced dynamics of $\mathcal{S}$ in the Heisenberg picture. The family $\left\{T_{\lambda}^{t}\right\}_{t \geq 0}$ is not a semigroup. However, one expects that $T_{\lambda}^{t / \lambda^{2}}$ converges to a semigroup as $\lambda \rightarrow 0$. This limiting semigroup describes the dynamics of open quantum system $\mathcal{S}$ in the Van Hove weak coupling limit.

For our purposes, the only important thing is that the Van Hove weak coupling limit exists and particular conditions which quarantee the existence of the limit are inessential. Hence, we postulate:

Assumption 2.A There exists an operator $\Gamma_{\mathrm{H}}: \mathcal{B}(\mathcal{K}) \rightarrow \mathcal{B}(\mathcal{K})$ such that for $t \geq 0$,

$$
\lim _{\lambda \rightarrow 0} P_{\mathrm{H}} \tau_{0}^{-t / \lambda^{2}} \tau_{\lambda}^{t / \lambda^{2}} P_{\mathrm{H}}=\mathrm{e}^{\mathrm{i} t \Gamma_{\mathrm{H}}} .
$$

We will call the operator $\Gamma_{\mathrm{H}}$ the Davies generator in the Heisenberg picture. A Fermi Golden Rule computation yields that

$$
\Gamma_{\mathrm{H}}=\lim _{\epsilon \nearrow 0} \sum_{e \in \operatorname{sp}([K,])} 1_{e}([K, \cdot])([V, \cdot])\left(e+\mathrm{i} \epsilon-\left[L_{0}^{\mathrm{semi}}, \cdot\right]\right)^{-1}([V, \cdot]) 1_{e}([K, \cdot])
$$

$\left(\operatorname{sp}(A)\right.$ stands for the spectrum of the operator $A$ and $1_{e}(A)$ for the spectral projection onto $e \in \operatorname{sp}(A)$ ), and indeed one can prove this formula under very general conditions (see [Da1, DJ3]). However, the specific form of $\Gamma_{\mathrm{H}}$ will not concern us here.

In the last several years there has been a revival of interest in rigorous mathematical study of the models $\left(\mathfrak{M}, \tau_{\lambda}\right)$. These studies were based on mathematical techniques (Tomita-Takesaki modular theory, quantum Koopmanism, Mourre theory, spectral 
complex deformations) which allowed for detailed understanding of the dynamics. The emerging picture is that ergodic properties and thermodynamics of the system $\mathcal{S}+\mathcal{R}$ are controlled by spectral resonances of two operators, the standard Liouvillean and CLiouvillean, canonically associated to the pair $\left(\mathfrak{M}, \tau_{\lambda}\right)$ by Tomita-Takesaki modular theory [JP1, JP2, JP4, JP5]. A natural and important question is how is the spectral Fermi Golden Rule for these resonances related to the generator $\Gamma_{\mathrm{H}}$. To describe the answer we will consider separately the thermal equilibrium and the nonequilibrium case.

\section{Thermal equilibrium case}

We will freely use the language and notation of algebraic quantum statistical mechanics and Tomita-Takesaki modular theory. The books [BR1, BR2, Ha, St, StZs] are standard references. A modern exposition can be also found in the recent article [DJP].

In this section the distinguished invariant state $\mathfrak{M}_{\mathcal{R}} \ni A \mapsto\left(\Omega_{\mathcal{R}} \mid A \Omega_{\mathcal{R}}\right)$ is a $\left(\tau_{\mathcal{R}}, \beta\right)$ KMS state for some $\beta>0$ (in other words, the reservoir is initially in thermal equilibrium at inverse temperature $\beta$ ).

The inner product $(X \mid B)=\operatorname{Tr}\left(X^{*} B\right)$ makes $\mathcal{B}(\mathcal{K})$ into Hilbert space, denoted $l^{2}(\mathcal{K})$. Note that $\mathcal{B}(\mathcal{K})$ acts naturally on $l^{2}(\mathcal{K})$ by right multiplication. This defines a representation $\pi_{\mathcal{S}}: \mathcal{B}(\mathcal{K}) \rightarrow \mathcal{B}\left(l^{2}(\mathcal{K})\right)$. Let $J_{\mathcal{S}}: l^{2}(\mathcal{K}) \rightarrow l^{2}(\mathcal{K})$ be defined by $J_{\mathcal{S}}(X)=X^{*}$, and let $l_{+}^{2}(\mathcal{K})$ be the set of all positive $X \in l^{2}(\mathcal{K})$. The algebra $\pi_{\mathcal{S}}(\mathcal{B}(\mathcal{K}))$ is in standard form on the Hilbert space $l^{2}(\mathcal{K})$, and its natural cone and modular conjugation are $l_{+}^{2}(\mathcal{K})$ and $J_{\mathcal{S}}$.

The representation $\pi_{\mathcal{S}}$ extends to a representation $\pi: \mathfrak{M} \rightarrow \mathcal{B}\left(l^{2}(\mathcal{K}) \otimes \mathcal{H}_{\mathcal{R}}\right)$ by

$$
\pi(B \otimes C)=\pi_{\mathcal{S}}(B) \otimes C .
$$

The von Neumann algebra $\pi(\mathfrak{M})$ is in standard form on the Hilbert space $l^{2}(\mathcal{K}) \otimes \mathcal{H}_{\mathcal{R}}$. The natural cone and the modular conjugation are

$$
\mathcal{H}^{+}=l_{+}^{2}(\mathcal{K}) \otimes \mathcal{H}_{\mathcal{R}}^{+}, \quad J=J_{\mathcal{S}} \otimes J_{\mathcal{R}}
$$

The standard Liouvillean, $L_{\lambda}$, is the unique selfadjoint operator on $l^{2}(\mathcal{K}) \otimes \mathcal{H}_{\mathcal{R}}$ such that

$$
\pi\left(\tau_{\lambda}^{t}(A)\right)=\mathrm{e}^{\mathrm{i} t L_{\lambda}} \pi(A) \mathrm{e}^{-\mathrm{i} t L_{\lambda}}, \quad \mathrm{e}^{\mathrm{i} t L_{\lambda}} \mathcal{H}^{+}=\mathcal{H}^{+} .
$$

( $L_{\lambda}$ implements the dynamics in the representation $\pi$ and preserves the natural cone). One easily shows that

$$
L_{\lambda}=L_{0}+\lambda \pi(V)-\lambda J \pi(V) J,
$$

where

$$
L_{0}=[K, \cdot] \otimes 1+1 \otimes L_{\mathcal{R}}
$$

see e.g. [DJP]. 
Consider the projection $\left.P_{\mathrm{L}}:=1 \otimes \mid \Omega_{\mathcal{R}}\right)\left(\Omega_{\mathcal{R}} \mid\right.$ on the Hilbert space $l^{2}(\mathcal{K}) \otimes \mathcal{H}_{\mathcal{R}}$. We identify $l^{2}(\mathcal{K})$ with $\operatorname{Ran} P_{\mathrm{L}}$ by $l^{2}(\mathcal{K}) \ni B \rightarrow B \otimes \Omega_{\mathcal{R}}$. Obviously, for $X, B \in l^{2}(\mathcal{K})$,

$$
\left(X \otimes \Omega_{\mathcal{R}} \mid \mathrm{e}^{-\mathrm{i} t L_{0}} \mathrm{e}^{\mathrm{i} t L_{\lambda}} B \otimes \Omega_{\mathcal{R}}\right)=\operatorname{Tr}_{\mathcal{K}}\left(X^{*} P_{\mathrm{L}} \mathrm{e}^{-\mathrm{i} t L_{0}} \mathrm{e}^{\mathrm{i} t L_{\lambda}} P_{\mathrm{L}} B\right) .
$$

We again postulate existence of the Van Hove limit.

Assumption 3.A There exists an operator $\Gamma_{\mathrm{L}}: l^{2}(\mathcal{K}) \rightarrow l^{2}(\mathcal{K})$ such that for $t \geq 0$,

$$
\lim _{\lambda \rightarrow 0} P_{\mathrm{L}} \mathrm{e}^{-\mathrm{i} t L_{0} / \lambda^{2}} \mathrm{e}^{\mathrm{i} t L_{\lambda} / \lambda^{2}} P_{\mathrm{L}}=\mathrm{e}^{\mathrm{i} t \Gamma_{\mathrm{L}}} .
$$

A Fermi Golden Rule computation yields that

$$
\begin{array}{r}
\left.\Gamma_{\mathrm{L}}=\lim _{\epsilon \nearrow 0} \sum_{e \in \operatorname{sp}([K, \cdot])} 1_{e}([K, \cdot])\right)(\pi(V)-J \pi(V) J) \\
\left(e+\mathrm{i} \epsilon-L_{0}\right)^{-1}(\pi(V)-J \pi(V) J) 1_{e}([K, \cdot]),
\end{array}
$$

and indeed one can prove this formula under very general conditions [Da1, DJ3]. The operator $\Gamma_{\mathrm{L}}$ is called the Level Shift Operator for the standard Liouvillean. The operator $[K, \cdot]+\lambda^{2} \Gamma_{\mathrm{L}}$ predicts location of eigenvalues and resonances of $L_{\lambda}$ to the 2nd order of perturbation theory and has been an important tool in the recent works on return to equilibrium [BFS, DJ1, DJ2, JP1, JP5, M].

We are interested in relation between $\Gamma_{\mathrm{L}}$ and the Davies generator $\Gamma_{\mathrm{H}}$. Obviously, as algebras, $\mathcal{B}(\mathcal{K}) \equiv l^{2}(\mathcal{K})=: \mathcal{V}$. Let $\gamma: \mathcal{V} \rightarrow \mathcal{V}$ be the linear invertible map defined by

$$
\gamma(B):=B \mathrm{e}^{-\beta K / 2} .
$$

Theorem 3.1 Assumption 2.A holds if and only if Assumption 3.A holds. If the assumptions hold, then

$$
\Gamma_{\mathrm{H}}=\gamma^{-1} \circ \Gamma_{\mathrm{L}} \circ \gamma
$$

Remark. Explicitely, for $B \in \mathcal{B}(\mathcal{K}) \equiv l^{2}(\mathcal{K})$, we have $\Gamma_{\mathrm{H}}(B)=\Gamma_{\mathrm{L}}\left(B \mathrm{e}^{-\beta K / 2}\right) \mathrm{e}^{\beta K / 2}$.

Proof. The Araki perturbation theory [BR2, DJP] yields that

$$
\Psi_{0}:=\mathrm{e}^{-\beta K / 2} \otimes \Omega_{\mathcal{R}} \in \operatorname{Dom}\left(\mathrm{e}^{-\beta\left(L_{0}+\lambda \pi(V)\right) / 2}\right),
$$

that the vector

$$
\Psi_{\lambda}:=\mathrm{e}^{-\beta\left(L_{0}+\lambda \pi(V)\right) / 2} \Psi_{0}
$$


belongs to $\operatorname{Ker} L_{\lambda}$, and that $\Psi_{\lambda}=\Psi_{0}+O(\lambda)$. For $X, B \in \mathcal{B}(\mathcal{K}) \equiv l^{2}(\mathcal{K})$ we have

$$
\begin{aligned}
& \operatorname{Tr}_{\mathcal{K} \otimes \mathcal{H}_{\mathcal{R}}}\left(X \otimes \mid \Omega_{\mathcal{R}}\right)\left(\Omega_{\mathcal{R}} \mid \tau_{0}^{-t} \tau_{\lambda}^{t}(B \otimes 1)\right)=\left(X^{*} \otimes \Omega_{\mathcal{R}} \mid \mathrm{e}^{-\mathrm{i} t L_{0}} \mathrm{e}^{\mathrm{i} t L_{\lambda}}(B \otimes 1) \mathrm{e}^{-\mathrm{i} t L_{\lambda}} \mathrm{e}^{\mathrm{i} t L_{0}} 1 \otimes \Omega_{\mathcal{R}}\right) \\
& =\operatorname{Tr}_{\mathcal{K}}\left(X P_{\mathrm{L}} \mathrm{e}^{-\mathrm{i} t L_{0}} \mathrm{e}^{\mathrm{i} t L_{\lambda}}\left(\pi_{\mathcal{S}}(B) \otimes 1\right) \mathrm{e}^{-\mathrm{i} t L_{\lambda}} \mathrm{e}^{\mathrm{i} t L_{0}} 1 \otimes \Omega_{\mathcal{R}}\right) \\
& =\operatorname{Tr}_{\mathcal{K}}\left(\mathrm{e}^{\beta K / 2} X\left[P_{\mathrm{L}} \mathrm{e}^{-\mathrm{i} t L_{0}} \mathrm{e}^{\mathrm{i} t L_{\lambda}}\left(\pi_{\mathcal{S}}(B) \otimes 1\right) \mathrm{e}^{-\mathrm{i} t L_{\lambda}} \mathrm{e}^{\mathrm{i} t L_{0}} 1 \otimes \Omega_{\mathcal{R}}\right] \mathrm{e}^{-\beta K / 2}\right) \\
& =\left(X^{*} \mathrm{e}^{\beta K / 2} \otimes \Omega_{\mathcal{R}} \mid\left(\pi_{\mathcal{S}}\left(P_{\mathrm{L}} \mathrm{e}^{-\mathrm{i} t L_{0}} \mathrm{e}^{\mathrm{i} t L_{\lambda}}\left(\pi_{\mathcal{S}}(B) \otimes 1\right) \mathrm{e}^{-\mathrm{i} t L_{\lambda}} \mathrm{e}^{\mathrm{i} t L_{0}} 1 \otimes \Omega_{\mathcal{R}}\right) \otimes 1\right) \mathrm{e}^{-\beta K / 2} \otimes \Omega_{\mathcal{R}}\right) \\
& =\left(X^{*} \mathrm{e}^{\beta K / 2} \otimes \Omega_{\mathcal{R}} \mid \mathrm{e}^{-\mathrm{i} t L_{0}} \mathrm{e}^{\mathrm{i} t L_{\lambda}}\left(\pi_{\mathcal{S}}(B) \otimes 1\right) \mathrm{e}^{-\mathrm{i} t L_{\lambda}} \mathrm{e}^{\mathrm{i} t L_{0}} \mathrm{e}^{-\beta K / 2} \otimes \Omega_{\mathcal{R}}\right) \\
& =\left(X^{*} \mathrm{e}^{\beta K / 2} \otimes \Omega_{\mathcal{R}} \mid \mathrm{e}^{-\mathrm{i} t L} \mathrm{e}^{\mathrm{i} t L_{\lambda}}(B \otimes 1) \mathrm{e}^{-\beta K / 2} \otimes \Omega_{\mathcal{R}}\right)+O(\lambda) \\
& =\left(X^{*} \mathrm{e}^{\beta K / 2} \otimes \Omega_{\mathcal{R}} \mid P_{\mathrm{L}} \mathrm{e}^{-\mathrm{i} t L} \mathrm{e}^{\mathrm{i} t L_{\lambda}} P_{\mathrm{L}} B \mathrm{e}^{-\beta K / 2} \otimes \Omega_{\mathcal{R}}\right)+O(\lambda) \\
& =\operatorname{Tr}_{\mathcal{K}}\left(X\left[P_{\mathrm{L}} \mathrm{e}^{-\mathrm{i} t L_{0}} \mathrm{e}^{\mathrm{i} t L_{\lambda}} P_{\mathrm{L}} B \mathrm{e}^{-\beta K / 2}\right] \mathrm{e}^{\beta K / 2}\right)+O(\lambda)
\end{aligned}
$$

uniformly for $t \geq 0$. Hence, for $X, B \in \mathcal{B}(\mathcal{K}) \equiv l^{2}(\mathcal{K})$,

$$
\operatorname{Tr}_{\mathcal{K}}\left(X P_{\mathrm{H}} \tau_{0}^{-t} \tau_{\lambda}^{t} P_{\mathrm{H}} B\right)=\operatorname{Tr}_{\mathcal{K}}\left(X\left[P_{\mathrm{L}} \mathrm{e}^{-\mathrm{i} t L_{0}} \mathrm{e}^{\mathrm{i} t L_{\lambda}} P_{\mathrm{L}} B \mathrm{e}^{-\beta K / 2}\right] \mathrm{e}^{\beta K / 2}\right)+O(\lambda)
$$

uniformly for $t \geq 0$. Since $\operatorname{dim} \mathcal{K}<\infty$, we conclude that for a given $t$ the limit

$$
\lim _{\lambda \rightarrow 0} P_{\mathrm{L}} \mathrm{e}^{-\mathrm{i} t L_{0} / \lambda^{2}} \mathrm{e}^{\mathrm{i} t L_{\lambda} / \lambda^{2}} P_{\mathrm{L}}=: T_{\mathrm{L}}^{t}
$$

exists iff the limit

$$
\lim _{\lambda \rightarrow 0} P_{\mathrm{H}} \tau_{0}^{-t / \lambda^{2}} \tau_{\lambda}^{t / \lambda^{2}} P_{\mathrm{H}}=: T_{\mathrm{H}}^{t}
$$

exists. Moreover, if the limits exist, then

$$
T_{\mathrm{H}}^{t}=\gamma^{-1} \circ T_{\mathrm{L}}^{t} \circ \gamma
$$

In particular, $T_{\mathrm{H}}^{t}$ is a semigroup iff $T_{\mathrm{L}}^{t}$ is a semigroup and their generators $\left(\Gamma_{\mathrm{H}}\right.$ and $\Gamma_{\mathrm{L}}$ respectively) satisfy (3.13).

\section{Nonequilibrium case}

We now consider the case where the reservoir is not in thermal equilibrium, namely where the invariant state

$$
\mathfrak{M}_{\mathcal{R}} \ni A \mapsto\left(\Omega_{\mathcal{R}} \mid A \Omega_{\mathcal{R}}\right)
$$


is not a $\left(\tau_{\mathcal{R}}, \beta\right)$-KMS state for any $\beta$. A typical example is a free Bose or Fermi gas with quasi-free initial state whose energy density is different from Planck's law. Another example is a multithermal reservoir where

$$
\begin{aligned}
\mathfrak{M}_{\mathcal{R}} & =\mathfrak{M}_{\mathcal{R}_{1}} \otimes \cdots \otimes \mathfrak{M}_{\mathcal{R}_{M}} \\
\tau_{\mathcal{R}} & =\tau_{\mathcal{R}_{1}} \otimes \cdots \otimes \tau_{\mathcal{R}_{M}} \\
\Omega_{\mathcal{R}} & =\Omega_{\mathcal{R}_{1}} \otimes \cdots \otimes \Omega_{\mathcal{R}_{M}}
\end{aligned}
$$

$\mathfrak{M}_{\mathcal{R}_{k}} \ni A \mapsto\left(\Omega_{\mathcal{R}_{k}} A \Omega_{\mathcal{R}_{k}}\right)$ is a $\left(\tau_{\mathcal{R}_{k}}, \beta_{k}\right)$-KMS state for some $\beta_{k}>0$, and not all $\beta_{k}$ are the same. This case has attracted considerable attention in the recent literature on nonequilibrium quantum statistical mechanics.

The standard Liouvillean is again well defined and given by (3.8). However, in nonequlibrium case and for $\lambda \neq 0, L_{\lambda}$ typically will have no point spectra. In particular, zero will not be an eigenvalue of $L_{\lambda}$. We recall that $\operatorname{Ker} L_{\lambda}=\{0\}$ iff $W^{*}$-dynamical system $\left(\mathfrak{M}, \tau_{\lambda}\right)$ has no normal, invariant states. Hence, in nonequilibrium case one expects that $\Gamma_{\mathrm{L}}$ will have no real eigenvalues and hence that $\Gamma_{\mathrm{H}}$ and $\Gamma_{\mathrm{L}}$ are not isospectral. In fact, in nonequilibrium case one expects no direct relation between $\Gamma_{\mathrm{H}}$ and $\Gamma_{\mathrm{L}}$.

The spectral approach to nonequilibrium quantum statistical mechanics has been recently proposed in [JP4]. The basic object is a non-selfadjoint generator of dynamics called C-Liouvillean. This operator is defined as follows.

Assume that $\Omega_{\mathcal{R}}$ is a cyclic (and hence separating) vector for $\mathfrak{M}_{\mathcal{R}}$ and let $\Delta$ be the corresponding modular operator. We assume that the operator

$$
\left(1 \otimes \Delta^{1 / 2}\right) \pi(V)\left(1 \otimes \Delta^{-1 / 2}\right)
$$

initially defined on $\mathfrak{M} 1 \otimes \Omega_{\mathcal{R}} \subset l^{2}(\mathcal{K}) \otimes \mathcal{H}_{\mathcal{R}}$, extends to an element of $\mathfrak{M}$. We denote this element by $\pi(V)_{\Delta}$ and set $\mathfrak{L}_{0}:=L_{0}$,

$$
\mathfrak{L}_{\lambda}=\mathfrak{L}_{0}+\lambda \pi(V)-\lambda J \pi(V)_{\Delta} J
$$

The operator $\mathfrak{L}_{\lambda}$ is called the C-Liouvillean of the system $\mathcal{S}+\mathcal{R}$. Note that except in trivial cases $\pi(V)_{\Delta}$ is not self-adjoint, and hence $\mathfrak{L}_{\lambda}$ is also not self-adjoint. Note also that $\mathfrak{L}_{\lambda}$ generates a $C_{0}$-semigroup on $l^{2}(\mathcal{K}) \otimes \mathcal{H}_{\mathcal{R}}$, that $\mathfrak{L}_{\lambda}\left(1 \otimes \Omega_{\mathcal{R}}\right)=1 \otimes \Omega_{\mathcal{R}}$, and that for all $A \in \mathfrak{M}$,

$$
\pi\left(\tau_{\lambda}^{t}(A)\right) 1 \otimes \Omega_{\mathcal{R}}=\mathrm{e}^{\mathrm{i} t \mathfrak{L}_{\lambda}} \pi(A) 1 \otimes \Omega_{\mathcal{R}}
$$

see [JP4] for details.

Assumption 4.A There exists an operator $\Gamma_{\mathrm{C}}: l^{2}(\mathcal{K}) \rightarrow l^{2}(\mathcal{K})$ such that for $t \geq 0$,

$$
\lim _{\lambda \rightarrow 0} P_{\mathrm{L}} \mathrm{e}^{-\mathrm{i} t \mathfrak{L}_{0} / \lambda^{2}} \mathrm{e}^{\mathrm{i} t \mathfrak{L}_{\lambda} / \lambda^{2}} P_{\mathrm{L}}=\mathrm{e}^{\mathrm{i} t \Gamma_{\mathrm{C}}} .
$$


A Fermi Golden Rule computation yields

$$
\begin{array}{r}
\Gamma_{\mathrm{C}}=\lim _{\epsilon \nearrow_{0}} \sum_{e \in \operatorname{sp}([K, \cdot])} 1_{e}([K, \cdot])\left(\pi(V)-J \pi(V)_{\Delta} J\right) \\
\left(e+\mathrm{i} \epsilon-\mathfrak{L}_{0}\right)^{-1}\left(\pi(V)-J \pi(V)_{\Delta} J\right) 1_{e}([K, \cdot]),
\end{array}
$$

and one can prove this formula under very general conditions [DJ3]. As expected, the operator $[K, \cdot]+\lambda^{2} \Gamma_{\mathrm{C}}$ predicts the location of resonances of $\mathfrak{L}_{\lambda}$ to the second order of perturbation theory [JP4]. The operator $\Gamma_{\mathrm{C}}$ is called the Level Shift Operator for the C-Liouvillean.

Theorem 4.1 Assumption 2.A holds if and only if Assumption 4.A holds. If the assumptions hold, then

$$
\Gamma_{\mathrm{H}}=\Gamma_{\mathrm{C}}
$$

Proof: The identities

$$
\begin{aligned}
\operatorname{Tr}_{\mathcal{K}}\left(X P_{\mathrm{L}} \tau_{0}^{-t} \tau_{\lambda}^{t} P_{\mathrm{L}}\right) & =\operatorname{Tr}_{\mathcal{K} \otimes \mathcal{H}_{\mathcal{R}}}\left(X \otimes \mid \Omega_{\mathcal{R}}\right)\left(\Omega_{\mathcal{R}} \mid \tau_{0}^{-t} \tau_{\lambda}^{t}(B \otimes 1)\right) \\
& =\left(X^{*} \otimes \Omega_{\mathcal{R}} \mid \mathrm{e}^{-\mathrm{i} t \mathfrak{L}_{0}} \mathrm{e}^{\mathrm{i} t \mathfrak{L}_{\lambda}}\left(\pi_{\mathcal{S}}(B) \otimes 1\right) 1 \otimes \Omega_{\mathcal{R}}\right) \\
& =\left(X^{*} \otimes \Omega_{\mathcal{R}} \mid \mathrm{e}^{-\mathrm{i} t \mathfrak{L}_{0}} \mathrm{e}^{\mathrm{i} t \mathfrak{L}_{\lambda}} B \otimes \Omega_{\mathcal{R}}\right) \\
& =\operatorname{Tr}_{\mathcal{K}}\left(X P_{\mathrm{L}} \mathrm{e}^{-\mathrm{i} t \mathfrak{L}_{0}} \mathrm{e}^{\mathrm{i} t \mathfrak{L}_{\lambda}} P_{\mathrm{L}}\right)
\end{aligned}
$$

yield that

$$
P_{\mathrm{H}} \tau_{0}^{-t} \tau^{t} P_{\mathrm{H}}=P_{\mathrm{L}} \mathrm{e}^{-\mathrm{i} t \mathfrak{L}_{0}} \mathrm{e}^{\mathrm{i} t \mathfrak{L}_{\lambda}} P_{\mathrm{L}}
$$

and the result is immediate.

We remark that C-Liouvillean is also well-defined in the thermal equilibrium and that in this case

$$
\mathfrak{L}_{\lambda}=\mathrm{e}^{\beta\left(L_{0}+\lambda \pi(V)\right) / 2} L_{\lambda} \mathrm{e}^{-\beta\left(L_{0}+\lambda \pi(V)\right) / 2},
$$

see [JP4]. Theorem 3.1 can be also proven using relations (4.19) and (4.20) and the argument of Section 5.6 in [DJP].

\section{Some remarks}

Theorem 3.1 extends to a large class of unbounded perturbations $V$. All what is needed is that $\left(\mathfrak{M}, \tau_{\lambda}\right)$ and $L_{\lambda}$ are well-defined and that the basic results of Araki's perurbation theory hold. The recent result [DJP] gives a set of sufficient conditions. Consider an 
unbounded self-adjoint operator $V$ on $\mathcal{K} \otimes \mathcal{H}_{\mathcal{R}}$ and assume:

(1) $V$ is affiliated with $\mathfrak{M}$.

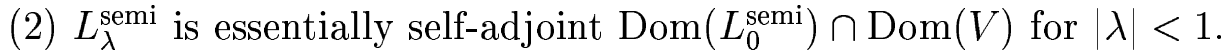

(3) $L_{\lambda}$ is essentially self-adjoint on $\operatorname{Dom}\left(L_{0}\right) \cap \operatorname{Dom}(\pi(V)) \cap \operatorname{Dom}(J \pi(V) J)$ for $|\lambda|<1$.

(4) $\left\|\mathrm{e}^{-\beta \lambda \pi(V) / 2} \Psi_{0}\right\|<\infty$ for $|\lambda|<1$.

Then the results of [DJP] yield that Theorem 3.1 holds with the same proof for the unbounded perturbation $V$. In particular, Theorem 3.1 holds for Pauli-Fierz systems with bosonic reservoirs.

The proof of Theorem 4.1 requires no estimates and follows from the identity (4.19). Obviously, this theorem holds whenever C-Liouvillean can be meaningfully defined, see [DJ3].

The results of this note bridge the gap between the large body of literature on Markovian semigroups for open quantum systems and the recent investigations of open quantum systems based on algebraic and spectral techniques. The main objects of the two approaches - the Davies generator and the Level Shift Operator for the standard and CLiouvillean - determine each other and hence the results of one approach can be used in the context of the other. This link is exploited in detail in the forthcoming article [DJ3].

Finally, we mention an early work [JP3] where the relation between $\Gamma_{\mathrm{H}}$ and $\Gamma_{\mathrm{L}}$ has been studied. In this work one can find an algorithm how to construct $\Gamma_{\mathrm{H} / \mathrm{L}}$ from $\Gamma_{\mathrm{L} / \mathrm{H}}$. This algorithm can be also used to prove Theorem 4.1 [Pi]. The direct proof of Theorem 3.1 given in this note is however considerably simpler.

\section{References}

[BR1] Brattelli, O., Robinson, D. W.: Operator Algebras and Quantum Statistical Mechanics, Volume 1. Springer-Verlag, Berlin, second edition 1987.

[BR2] Brattelli, O., Robinson, D. W.: Operator Algebras and Quantum Statistical Mechanics, Volume 2. Springer-Verlag, Berlin, second edition 1996.

[BFS] Bach, V., Fröhlich, J., Sigal, I.: Return to equilibrium. J. Math. Phys. 41, 3985 (2000).

[Da1] Davies, E. B.: Markovian master equations. Commun. Math. Phys. 39, 91 (1974).

[Da2] Davies, E. B.: Markovian master equations II. Math. Ann. 219, 147 (1976). 
[Da3] Davies, E. B.: One parameter semigroups, Academic Press 1980

[DJ1] Dereziński, J., Jakšić, V.: Spectral theory of Pauli-Fierz operators, J. Func. Anal. 180, 241 (2001).

[DJ2] Dereziński, J., Jakšić, V.: Return to equilibrium for Pauli-Fierz systems. To appear in Ann. Henri Poincaré.

[DJ3] Dereziński, J., Jakšić, V.: In preparation.

[DJP] Dereziński, J., Jakšić, V., Pillet, C. A.: Perturbation theory of $W^{*}$-dynamics, Liouvilleans and KMS-states. To appear in Rev. Math. Phys.

[F] Fermi, E.: Nuclear physics. Notes compiled by Orear J., Rosenfeld A.H. and Schluter R.A. The University of Chicago Press, Chicago, 1950.

[GFVKS] Gorini, V., Frigerio, A., Verri, M., Kossakowski, A., Sudarshan, E,C.G.: Properties of quantum markovian master equations. Rep. Math. Phys. 13, 149 (1978).

[Ha] Haag, R.: Local quantum physics. Springer-Verlag, Berlin, 1993.

[Haa] Haake, F.: Statistical treatment of open systems by generalized master equation. Springer Tracts in Modern Physics 66, Springer-Verlag, Berlin, 1973.

[JP1] Jakšić, V., Pillet, C.-A.: On a model for quantum friction III. Ergodic properties of the spin-boson system. Commun. Math. Phys. 178, 627 (1996).

[JP2] Jakšić, V., Pillet, C.-A.: Spectral theory of thermal relaxation. J. Math. Phys. 38, 1757 (1997).

[JP3] Jakšić, V., Pillet, C.-A.: From resonances to master equations. Ann. Inst. Henri Poincaré 67, 425 (1997).

[JP4] Jakšić, V., Pillet, C.-A.: Non-equilibrium steady states for finite quantum systems coupled to thermal reservoirs. Commun. Math. Phys. 226, 131 (2002).

[JP5] Jakšić, V., Pillet, C.-A.: Mathematical theory of non-equilibrium quantum statistical mechanics. J. Stat. Phys. 108, 787 (2002).

[KTH] Kubo, R., Toda, M., Hashitsume, N.: Statistical Physics II. Nonequilibrium Statistical Mechanics. Springer-Verlag, Berlin, 1985.

[M] Merkli, M.: Positive commutators in non-equilibrium quantum statistical mechanics. Commun. Math. Phys. 223, 327 (2001).

[LeSp] Lebowitz, J., Spohn, H.: Irreversible thermodynamics for quantum systems weakly coupled to thermal reservoirs. Adv. Chem. Phys. 39, 109 (1978). 
[Pa1] Pauli, W.: Fesrshrift zum 60. Gerburstage A. Sommerfeld, S.30, Leipzig, Hirzel 1928.

[Pa2] Pauli, W.: Pauli Lectures on Physics: Volume 4. Statistical Mechanics. Edited by C.P. Enz, The MIT Press, Cambridge, 1973.

[Pi] Pillet, C.-A.: Private communication.

[Ru] Ruelle, D.: Natural nonequilibrium states in quantum statistical mechanics. J. Stat. Phys. 98, 57 (2000).

[St] Stratila, S.: Modular theory in operator algebras. Abacus Press, Turnbridge Wells, 1981.

[StZs] Stratila, S., Zsido, L.: Lectures on von Neumann algebras. Abacus Press, Turnbridge Wells, 1979.

[VH] Van Hove, L.: Master equation and approach to equilibrium for quantum systems. In Fundamental problems in statistical mechanics, combined by E.G.D. Cohen, North-Holand, Amsterdam 1962.

[W] Weisskopf, V., Wigner, E.P.: Berechnung der natürlichen Linienbreite auf Grund der Diracschen Lichttheorie. Z. Phys. 63, 54 (1930). 